\title{
Corrigendum: Induction, suppression and requirement of RNA silencing pathways in virulent Agrobacterium tumefaciens infections
}

Patrice Dunoyer, Christophe Himber \& Olivier Voinnet

Nat. Genet. 38, 258-263 (2006); published online 22 January 2006; corrected after print 26 June 2015

In the version of this article initially published, in Figure $5 \mathrm{c}$ (miR162) and Figure 5e (miR171), the rRNA images were duplicated without explanation. The figure legend should have stated that these two panels show the fluorescence image of the common rRNA loading control and autoradiographs of the same blot stripped and rehybridized with the two miRNAs, respectively. The authors state that all the display items in this publication and its Supplementary Information are previously unpublished work and accurate representations of the experiments described. The legend of Figure 5 should read:

Figure 5. RNA blot analysis of endogenous small RNAs in leaves, stems and tumors of A. thaliana. Cis-acting (a) and trans-acting (b) siRNAs. (c) Evolutionarily conserved miRNAs. Note that a 24 -nt species ${ }^{18}$ for miR156 and miR162 is eliminated in tumors. (d) A. thaliana-specific miR163. (e) Schematic of the GFP-based sensor construct reporting cleavage by the evolutionarily conserved miR171 (upper panel). RNA blot analysis of miR171 and of the GFP sensor mRNA (middle and lower panels, respectively). The middle panel of $\mathbf{e}$ and bottom panel of $\mathbf{c}$ show the rRNA and stripped and reprobed RNA blot for the same gel. (f) Accumulation of two evolutionarily conserved miRNAs targeting important positive regulators of the plant auxin response. L, leaf; S, stem; T, tumor; rRNA, ethidium bromide staining of ribosomal RNA. 